\title{
Catch the Best: Novel Screening Strategy to Select Stress Protecting Agents for Crop Plants
}

\section{Christin Zachow ${ }^{1,2, *}$, Henry Müller ${ }^{2}$, Ralf Tilcher ${ }^{3}$, Christina Donat ${ }^{4}$ and Gabriele Berg ${ }^{2}$}

1 Austrian Center of Industrial Biotechnology (ACIB GmbH), Petersgasse 14, 8010 Graz, Austria

2 Institute of Environmental Biotechnology, Graz University of Technology, Petersgasse 12, A-8010 Graz, Austria; E-Mails: henry.mueller@tugraz.at (H.M.); gabriele.berg@tugraz.at (G.B.)

3 KWS SAAT AG, Grimsehlstraße 31, D-37555, Einbeck, Germany; E-Mail: ralf.tilcher@kws.com

4 BIO-FERM GmbH, Technologiezentrum Tulln, Technopark 1 B, 3430 Tulln, Austria;

E-Mail: christina.donat@bio-ferm.com

* Author to whom correspondence should be addressed; E-Mail: christin.zachow@tugraz.at; Tel.: +43-316-873-8423; Fax: +43-316-873-8819.

Received: 23 August 2013; in revised form: 13 November 2013 / Accepted: 19 November 2013 / Published: 26 November 2013

\begin{abstract}
Climate change increases stress levels for crops and affects the economic and environmental aspects of agricultural management systems. The application of stress tolerance-mediating microorganisms is an auspicious strategy for improving crop protection, and as such, we developed a direct selection strategy to obtain cultivable microorganisms from promising bioresources using the bait plants, maize, oilseed rape, sorghum and sugar beet. Alpine mosses, lichens and primrose were selected as bioresources, as each is adapted to adverse environmental conditions. A 10\% crop-specific selection was found for bait plant rhizosphere communities using cultivation-independent fingerprints, and their potential role as stress protecting agents (SPA) was evaluated following the cultivation of captured bacteria. In addition to assays identifying phytopathogen antagonism and plant growth promotion capacities, our evaluation included those that test the ability to allocate nutrients. Moreover, we developed new assays to measure tolerance in diverse stress conditions. A score scheme was applied to select SPAs with desired properties, and three Pseudomonas species with pronounced antagonistic activity that showed elevated tolerance to desiccation and an improved seed germination rate were subsequently chosen. Screening for environmentally-conditioned and host-adapted microorganisms provides a novel tool for target-oriented exploitation of microbial bioresources for the management of ecofriendly crops facing biotic and abiotic stresses.
\end{abstract}


Keywords: bait plants; biological control agents (BCAs); stress protecting agents (SPAs); screening; abiotic stress

\section{Introduction}

The impact of climate change has had many dramatic and multifaceted ecological consequences on natural and human-managed systems, apart from the prevalent threat of phytopathogens and nutrient deficiency on monocultured crops. Plants are affected by increasing temperatures, salination, drought and solar radiation, and with these direct biological effects come rapidly evolving pathogens with shifting host and geographic distribution, resulting in reduced crop yield and quality [1-3]. The most effective targets for improved sustainability are those with tolerance traits for abiotic and biotic stresses that match with the associated microbiome, the so-called second plant genome [4]. For biological control of phytopathogens, beneficial microorganisms were developed as biological control agents (BCAs) to act as an alternative to conventional pesticides. However, to overcome stress conditions, stress protecting agents (SPAs) [5], which are microorganisms with the ability to mediate stress protection to the host plant and to survive under adverse abiotic conditions, are required.

The first step in our study was to develop an ecologically-based screening strategy for where to search for SPAs. Plants are associated with highly diverse functional microorganisms, which promote plant growth, health and stress tolerance [6]. In natural environments, microorganisms' versatility and ability to cope with harsh conditions is facilitated by various elaborate enzymes and sophisticated mechanisms. Thus, plants from natural environments can be a promising source to screen for bacteria and fungi with properties relevant to almost all fields of biotechnology. Today, numerous microbes initially isolated from plant habitats are employed in biotechnological processes, especially in agriculture [7,8]. Microorganisms associated with various plant microenvironments constitute a rich source for applications in biotechnological processes and sustainable agriculture. Thus, the rhizosphere and endosphere have been found as particularly interesting reservoirs for potential microorganisms [6] capable of promoting plant growth and inducing systemic tolerance towards salt and drought [9]. Recently, various species of lichens and mosses have emerged as potential bioresources for antagonistic and plant growth promoting (PGP) microorganisms [10-12]. Additionally, antagonistic microorganisms have been found to be associated with mistletoe species and sclerotia from Rhizoctonia solani [13,14]. Although the mentioned bioresources are well-known and interesting, it is still difficult to isolate microorganisms from these sources, because they need specific or currently unknown conditions for their growth in vitro. The development of biological plant protection products usually begins with the enrichment of microorganisms on artificial growth media. Regardless of the medium used for cultivation, two major problems arise. Firstly, only $1 \%$ to $10 \%$ of microbial species are able to grow on conventional media [15]. Secondly, faster growing microbes typically outgrow the slower ones. As a result, current cultivation techniques yield a low microbial diversity. Furthermore, most of the applied enrichment strategies neglect the conditions to which the selected microorganisms will be later applied. Superior growth under laboratory conditions does not necessarily guarantee a good performance ad planta. Therefore, a second question arose as to how we could gather suitable SPAs. 
The objective of this study was to use bait plants for a more directed selection of suitable microorganisms from stress-exposed bioresources. We used pathogen- and low pH-tolerant mosses [11], cold-adapted primrose [7,8] and drought-adapted lichens [12] as natural sources for microorganisms. The microorganisms from the original host were captured by priming the seeds of the bait plants, maize, oilseed rape, sorghum and sugar beet, and, subsequently, culturing the microbial community from the rhizosphere of each bait plant. The screening procedure was accompanied by microbial community fingerprinting using the single strand conformation polymorphism (SSCP) of 16S rRNA genes [16], which is useful for a fast and safe evaluation of microbial shifts [17]. Re-cultivated bacteria were intensively screened for their potential as biocontrol agents and stress protection mediators. The screening strategy included assays to identify: (1) antagonistic activity against diverse Ascomycota (Alternaria alternata, Botrytis cinerea, Sclerotium sclerotiorum, Verticillium dahliae), Basidiomycota (Rhizoctonia solani AG2-2IIIB, R. solani AG4, Sclerotium rolfsii) and the oomycete, Phytophthora infestans; (2) nutrient allocation (iron, nitrogen, phosphate); (3) exoenzyme activity (chitinase, glucanase, protease); (4) antibiotic genes (pyrrolnitrin prn $\mathrm{D}$, diacetylphloroglucinol phlD); (5) growth in the presence of diverse stress conditions (drought, reactive oxygen species, salt, temperature); (6) seed germination promotion; as well as (7) biosurfactant production. There is a general trend towards greater suppression or enhanced consistency of the antagonistic and stress protecting effect of microorganisms. Therefore, a scoring scheme was applied to specifically select desired properties as an example for the most suitable BCAs and SPAs in potential applications, and the resulting strategy provides a novel tool for target-oriented exploitation of bioresources.

\section{Results and Discussion}

\subsection{Bait Plant Strategy}

All microbial communities extracted from the field-collected moss, lichen and primrose from their natural environments were used to prime seeds of maize, oilseed rape, sorghum and sugar beet. After two weeks of cultivation in pots with standardized potting soil, the structure and function of the microbial community was analyzed with cultivation-dependent and -independent methods. The resulting bacterial isolates were assumed to be environmentally-conditioned and host-adapted (Figure 1).

\subsection{Insight into Rhizosphere Communities Using Molecular Fingerprints}

The bioresources were selected due to their potential to survive under particular adverse abiotic conditions. For example, Sphagnum mosses and their diverse microbiome undergo repetitive desiccation and oxidative stress. The bacterial capacity to tolerate oxidative stress, due to the high abundance of genes responsible for the oxidative stress response may determine the effective and stable colonization of the Sphagnum mosses [18]. Figure 2 shows microbial fingerprints of the moss-associated community captured by the bait plants. Interestingly, in each capture experiment, a portion of the moss-associated community was able to colonize the crops, and the bacterial community patterns from moss-treated crops showed host-specific communities. Bacteria derived from the moss community were specifically captured by the different crops; the bacterial communities captured through oilseed rape and sugar beet differed significantly ( $p \leq 0.0360, p \leq 0.0144$ ) from the other crops, and the captured communities of 
maize and sorghum differed at $p \leq 0.2980$ and $p \leq 0.1827$, respectively. Comparing bacterial community patterns from moss-microbiome-treated and non-treated control plants (sterile sodium chloride treatment), no significant differences were found. The microbial patterns resembled each other at a similarity level of 90\% (Figure 3). The highly similar microbial patterns observed for moss-treated crops compared to the controls showed that the bacteria added to the bait plants in target host treatments need to be both compatible and competitive within the already existing and established microbiome of the unsterile soil and seed community. In this way, microorganisms with antagonistic activity are typically known for their competitive nature and other beneficial properties, and it is thus more likely that they successfully establish in new environments [19]. As soil is the main reservoir for rhizosphere microorganisms [20], the plant host habitat and root exudates shape the soil bacterial community structure. A subset of the soil bacterial community is present around the plant roots, and active community members benefit from its basic functions and, in turn, help the plant thrive in specific environments [21,22]. Specific enrichment of microorganisms is already a standard in laboratory practices through the application of defined media; however, the application of natural bait material is a rarely applied technique in capturing potential target-adapted antagonists. Similar experiments were previously successfully applied, such as using Rhizoctonia solani sclerotia as bait material deposited in soil to enrich antagonistic counterparts [14]. The SSCP microbial fingerprint analysis was not suitable to detect bacterial populations derived from the treatments, as only the first one hundred dominant species are detectable. However, the most matching and stress protection mediating bacteria could be a part of the minor fraction of the microbial community. The importance of minor abundant microorganisms' function is gaining recognition in scientific research and discussion, and a better understanding is becoming possible, due to next generation sequencing methods that allow deeper insight into the microbial communities.

Figure 1. Selection strategy. Abbreviations: R2A, minimal medium to isolate universal bacteria; SSCP, single strand conformation polymorphisms analysis.

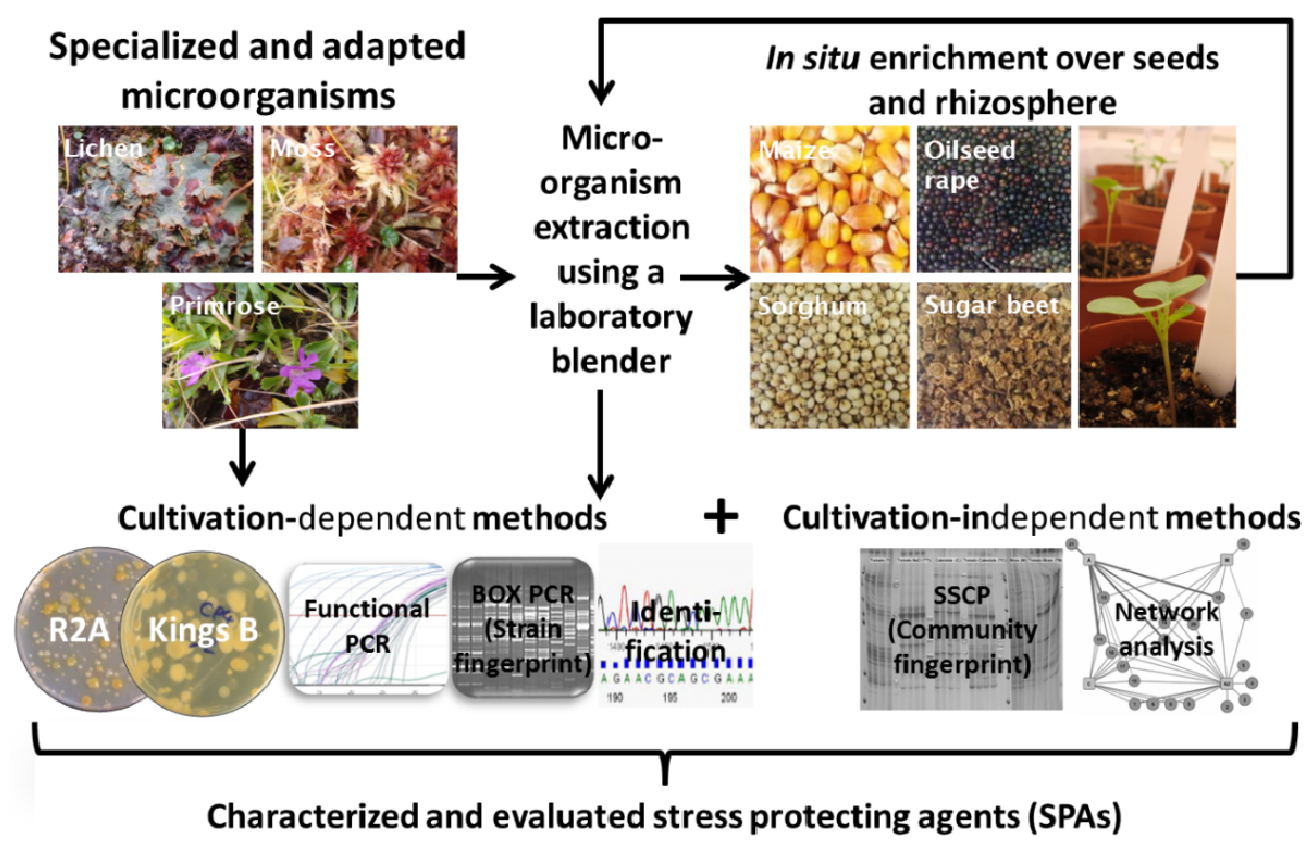


Figure 2. PCR-SSCP profiles of universal bacterial rhizosphere communities of moss-primed and untreated crop plants in two replicates (number) with three repetitions per plant species (three lanes per replicate). Legend: Std, standard $1 \mathrm{~kb}$ ladder for gel analysis. Internal marker: single bacterial isolates (right) and a mixture (middle) were sequenced and identified for the rhizosphere. Ladder maize (M): 1, Pseudomonas kilonensis KA13; 2, Pseudomonas brassicacearum subsp. brassicacearum KA15; 3, Arthrobacter nicotinovorans KA14. Ladder oilseed rape (R): 1, Pseudomonas brassicacearum subsp. brassicacearum KA36; 2, Pantoea sp. KA42; 3, Pseudomonas otitidis KA38. Ladder sorghum (S): 1, Pseudomonas veronii KA63. Ladder sugar beet (Z): 1, Rhodanobacter fulvus KA95; 2, Pseudomonas otitidis KA93; 3, Chryseobacterium wanjuense KA91; 4, Microbacterium xylanilyticum KA90. Control: crops were treated with sterile $0.85 \%$ sodium chloride solution.

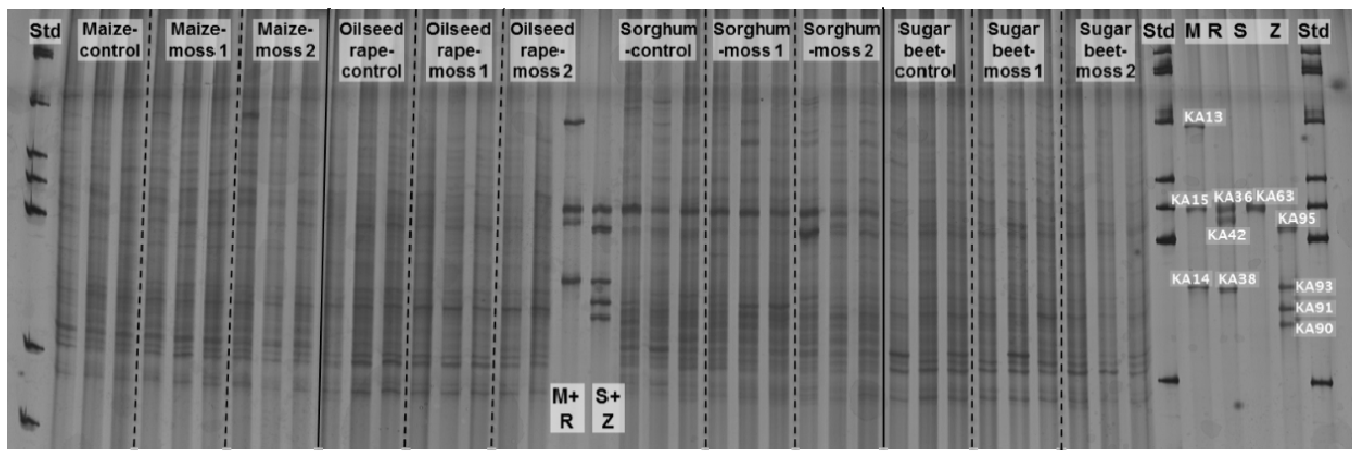

Figure 3. Unweighted pair group method with arithmetic mean (UPGMA) dendrogram of bacterial rhizosphere communities of moss-primed and untreated crop plants in two replicates (first number) with three repetitions per plant species (second number). The dendrograms were generated from SSCP community profiles using GelCompar II ${ }^{\circledR}$. The following settings were used: dendrogram type: UPGMA; similarity coefficient: curve-based Pearson correlation; optimization: 4\%; position tolerance: $1 \%$. The dashed line indicates a similarity level at $90 \%$. Letter code: first letter of the bait plant species; M, maize; $\mathrm{R}$, oilseed rape; S, sorghum; $\mathrm{Z}$, sugar beet; the second letter is the treatment; $\mathrm{M}$, moss; C, control.

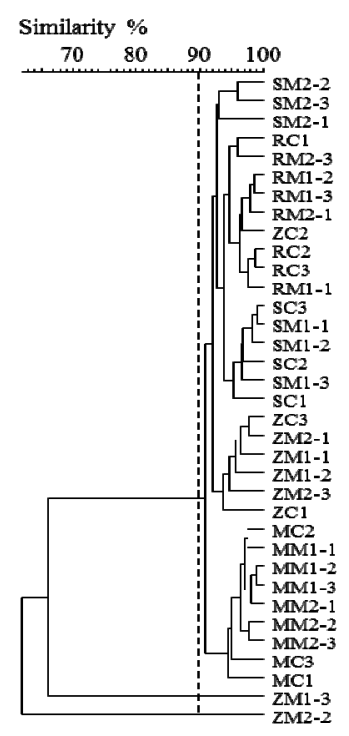




\subsection{Characterization of Selected Bacteria and Score Evaluation for SPAs}

In the first step, 283 microorganisms were isolated from the rhizospheres of bait plants. All bacteria antagonistic (105) against at least one of the tested pathogens, Alternaria alternata, Botrytis cinerea, Sclerotium sclerotiorum, Verticillium dahliae, Rhizoctonia solani AG2-2IIIB and AG4, Sclerotium rolfsii and Phytophthora infestans, were further characterized using various assays (Table 1). The screenings was comprised of parameters of: (1) nutrient allocation (iron, nitrogen, phosphate); (2) exoenzyme activity (chitinase, glucanase, protease); (3) antibiotic genes (pyrrolnitrin prnD, diacetylphloroglucinol phlD); (4) growth in the presence of diverse stress conditions (reactive oxygen species, drought, salt, temperature); (5) seed germination promotion; as well as (6) biosurfactant production; which were evaluated and weighted (one to five) according to their intended field application. Each parameter was defined with "one" for a positive reaction or maximum data range comparing values of all tested bacteria and "zero" as a negative or minimum data range. Positive values were multiplied with the defined evaluation weight, and all values were summarized to the final score. The higher the final score, the more the bacteria were assumed to be suitable for particular applications as SPAs. The weighted rating is adjustable in accordance to the target application. We defined a rating in order to yield the most potential antagonists with lytic and phosphate-solubilizing activity, which could efficiently enhance the mobilization of nutrients for themselves and for the associated host plant [12]. Additionally, detected genes that decode for antibiotic synthesis were excluded to advance other mechanisms involved in biocontrol, e.g., the production of volatiles. Due to elevated stress levels in crops as a result of climate change, the application of stress tolerance-mediating microorganisms is a new possibility to improve crop protection. Therefore, we highlighted bacteria that were able to grow in the presence of stresses, i.e., desiccation, low temperatures and oxidative agents. We valued protease production less, because it is a general mechanism and does not help differentiate between antagonists. Moreover, the score assists in the selection of bacteria for certain target applications, yet remains adjustable for different focuses. To address certain host plants, we set various values in plant growth promotion assays. Additionally, we differentiated on the basis of seed germination after three days and plant emergence after six days to evaluate the possible positive or negative effects of the applied bacteria. Only treatments comparable or better than the controls were highly weighted. The score of the isolates ranged from 10 (one isolate) to 47 (two isolates), whereas the majority shared the score of 32 (10) (Table 2). The three highest assessed bacteria, RM1-3-1 (KA38), SP2-1-2 (KA77) and MP1-3-2 (KA23), shared the score 47, 47 and 46, respectively. They are each a member of the genus, Pseudomonas, have pronounced antagonistic activity, can be re-cultivated after desiccation and improved the germination rate of seeds. Several of the analyzed parameters, e.g., antagonism, exoenzyme activity and PGP, are well known to characterize BCAs [23]. Plants and their microbiome are similarly influenced by environmental conditions with specific biotic and abiotic factors [6]. The novelties of this study are the directed selection by bait plants and, particularly, the application of diverse stress assays, which support the potential of BCAs to act as SPAs (parameter 5). Interestingly, the majority of the screened isolates could grow at low temperatures $(91.4 \%$ to $95.2 \%)$ and tolerated desiccation for $24 \mathrm{~h}(81.9 \%)$, yet only a few specialists were able to withstand oxidative stresses $(6.7 \%$ to $15.2 \%)$. 
Table 1. Scoring scheme. ${ }^{\text {a }}$ yes/no: positive (1) or negative (0) result. Range: high, 1; low, 0. Abbreviations: AB, antibiotic; TMAMQ, tetramethoxy azobismethylene quinone; ROS, reactive oxygen species; ONC, overnight culture.

\begin{tabular}{|c|c|c|c|c|}
\hline \multicolumn{3}{|l|}{ Screening Target } & \multirow{2}{*}{$\frac{\text { Result }^{\text {a }}}{\text { yes/no }}$} & \multirow{2}{*}{$\begin{array}{l}\text { Evaluation } \\
\text { Weight }\end{array}$} \\
\hline \multirow{8}{*}{ (1) Antagonism } & \multirow{4}{*}{ Ascomycota } & Alternaria alternata Nees & & \\
\hline & & Botrytis cinerea Pers. & yes/no & 0 \\
\hline & & Sclerotium sclerotiorum Sacc. & yes/no & 3 \\
\hline & & Verticillium dahliae Kleb. V25 & yes/no & 3 \\
\hline & \multirow{3}{*}{ Basidiomycota } & Rhizoctonia solani Kühn AG2-2IIIB & yes/no & 3 \\
\hline & & Rhizoctonia solani Kühn AG4 & yes/no & 3 \\
\hline & & Sclerotium rolfsii (Curzi) C.C.Tu and Kimbr. & yes/no & 3 \\
\hline & Oomycetes & Phytophthora infestans (Mont.) De Bary & yes/no & 3 \\
\hline \multirow{3}{*}{\multicolumn{2}{|c|}{ (2) Nutrient allocation }} & Nitrogen fixation & yes/no & 1 \\
\hline & & Phosphate solubilizing & yes/no & 3 \\
\hline & & Siderophore production & yes/no & 3 \\
\hline \multirow{3}{*}{\multicolumn{2}{|c|}{ (3) Exoenzyme activity }} & Chitinase & yes/no & 3 \\
\hline & & Glucanase & yes/no & 3 \\
\hline & & Protease & yes/no & 1 \\
\hline \multirow{2}{*}{\multicolumn{2}{|c|}{ (4) AB gene detection }} & Pyrrolnitrin (prnD) & yes/no & 0 \\
\hline & & Diacetylphloroglucinol ( $p h l \mathrm{D})$ & yes/no & 0 \\
\hline \multirow{9}{*}{$\begin{array}{l}\text { (5) Growth in } \\
\text { presence of stress }\end{array}$} & \multirow{3}{*}{$\begin{array}{l}\text { ROS detection } \\
\text { ROS }\end{array}$} & TMAMQ & Range & 5 \\
\hline & & Tellurite & Range & 3 \\
\hline & & $\mathrm{H}_{2} \mathrm{O}_{2}$ & Range & 3 \\
\hline & Drought & $24 \mathrm{~h}$ Desiccation & yes/no & 5 \\
\hline & Salt & In presence of various $\mathrm{NaCl}$ concentrations & Range & 3 \\
\hline & & $\mathrm{NA}, 24 \mathrm{~h}, 13^{\circ} \mathrm{C}$ & yes/no & 4 \\
\hline & Tamenomatuma & $\mathrm{NA}, 24 \mathrm{~h}, 37^{\circ} \mathrm{C}$ & yes/no & 1 \\
\hline & Iemperatures & $\mathrm{R} 2 \mathrm{~A}, 24 \mathrm{~h}, 13^{\circ} \mathrm{C}$ & yes/no & 4 \\
\hline & & $\mathrm{R} 2 \mathrm{~A}, 24 \mathrm{~h}, 37^{\circ} \mathrm{C}$ & yes/no & 1 \\
\hline \multirow{10}{*}{ (6) Plant assay } & \multirow{4}{*}{$\begin{array}{l}\text { Germination } \\
\text { after } 3 \text { days }\end{array}$} & Maize & Range & 3 \\
\hline & & Oilseed rape & Range & 1 \\
\hline & & Sorghum & Range & 1 \\
\hline & & Sugar beet & Range & 3 \\
\hline & \multirow{6}{*}{$\begin{array}{l}\text { Germination } \\
\text { after } 6 \text { days }\end{array}$} & Maize & Range & 1 \\
\hline & & Oilseed rape & Range & 1 \\
\hline & & Sorghum & Range & 3 \\
\hline & & Sugar beet & Range & 3 \\
\hline & & Indole-3-acetic acid & Range & 3 \\
\hline & & ACC deaminase & Range & 3 \\
\hline \multirow{5}{*}{ (7) Surface activity } & & Rhamnolipid & yes/no & 1 \\
\hline & & Toluol & yes/no & 1 \\
\hline & & Paraffin (ONC) & yes/no & 1 \\
\hline & & Engine oil (ONC) & yes/no & 1 \\
\hline & & Drop collapse assay & yes/no & 1 \\
\hline
\end{tabular}


Table 2. Score scheme results with maximum numbers of positive reactions for each characterization group. ${ }^{\text {a }}$ Strain number with color code: rhizosphere samples from maize, yellow/orange/red; oilseed rape, green shades; sorghum, blue shades; sugar beet, purple shades. The letter code is the first letter of the crop: M, maize; R, oilseed rape; S, sorghum; Z, sugar beet. The second letter is the treatment: F, lichen; M, moss; P, primrose. AB, antibiotic; n.d., not determined; ROS, reactive oxygen species. ARDRA, amplified ribosomal RNA gene restriction analysis.

\begin{tabular}{|c|c|c|c|c|c|c|c|c|c|c|c|}
\hline \multicolumn{4}{|c|}{ Strain information } & \multirow[b]{2}{*}{ Antagonism } & \multirow[b]{2}{*}{$\begin{array}{l}\text { Nutrient } \\
\text { allocation }\end{array}$} & \multirow[b]{2}{*}{$\begin{array}{c}\text { Exoenzyme } \\
\text { activity }\end{array}$} & \multirow[b]{2}{*}{$\begin{array}{l}\text { AB gene } \\
\text { detection }\end{array}$} & \multirow[b]{2}{*}{ Stress } & \multirow[b]{2}{*}{$\begin{array}{l}\text { Plant } \\
\text { assay }\end{array}$} & \multirow[b]{2}{*}{$\begin{array}{l}\text { Surface } \\
\text { activity }\end{array}$} & \multirow[b]{2}{*}{$\begin{array}{l}\text { Score } \\
\text { values }\end{array}$} \\
\hline Abb. & Species (risk group) & $\begin{array}{c}\text { Strain } \\
\text { number } \\
\end{array}$ & $\begin{array}{c}\text { ARDRA/ } \\
\text { BOX group }\end{array}$ & & & & & & & & \\
\hline KA001 & Pseudomonas kilonensis (1) & MF1-1-4 & $10 / 13$ & 6 & 2 & 1 & 0 & 5 & 4 & 0 & 43 \\
\hline KA002 & & MF1-2-3 & n.d./18 & 2 & 2 & 0 & 0 & 5 & 5 & 1 & 32 \\
\hline KA003 & Arthrobacter nitroguaiacolicus (1) & MF1-2-4 & $13 / 7$ & 2 & 2 & 2 & 1 & 6 & 2 & 1 & 37 \\
\hline KA004 & & MF1-3-1 & $10 / 13$ & 3 & 2 & 1 & 0 & 6 & 3 & 0 & 36 \\
\hline KA005 & & MF2-1-1 & $13 / 3$ & 3 & 0 & 1 & 0 & 6 & 3 & 1 & 35 \\
\hline KA006 & & MF2-1-2 & $10 / 13$ & 5 & 2 & 1 & 0 & 5 & 4 & 0 & 40 \\
\hline KA007 & & MF2-1-3 & $10 / 13$ & 5 & 2 & 1 & 0 & 5 & 3 & 0 & 39 \\
\hline KA008 & Pseudomonas corrugata (1) & MF2-3-1 & $10 / 13$ & 2 & 2 & 0 & 0 & 5 & 4 & 0 & 30 \\
\hline KA009 & Pseudomonas kilonensis (1) & MM1-2-2 & $10 / 13$ & 6 & 2 & 1 & 0 & 5 & 3 & 0 & 40 \\
\hline KA010 & & MM1-2-3 & $10 / 13$ & 6 & 2 & 1 & 0 & 5 & 2 & 0 & 41 \\
\hline KA011 & & MM1-2-4 & $10 / 13$ & 5 & 2 & 1 & 0 & 5 & 4 & 0 & 40 \\
\hline KA012 & & MM1-3-1 & $10 / 13$ & 5 & 2 & 1 & 0 & 5 & 3 & 0 & 39 \\
\hline KA013 & Pseudomonas kilonensis (1) & MM1-3-2 & $10 / 16$ & 6 & 2 & 1 & 0 & 5 & 3 & 0 & 40 \\
\hline KA014 & Arthrobacter nicotinovorans (1) & MM1-3-4 & $13 / 3$ & 2 & 0 & 1 & 0 & 5 & 2 & 5 & 27 \\
\hline KA015 & $\begin{array}{c}\text { Pseudomonas brassicacearum subsp. } \\
\text { brassicacearum (1) }\end{array}$ & MM2-1-2 & $10 / 23$ & 2 & 1 & 1 & 1 & 5 & 2 & 1 & 27 \\
\hline KA016 & & MM2-1-4 & $5 / 1$ & 4 & 1 & 1 & 1 & 6 & 1 & 3 & 37 \\
\hline KA017 & Pseudomonas kilonensis (1) & MM2-2-2 & $9 / 13$ & 4 & 1 & 0 & 0 & 2 & 1 & 3 & 24 \\
\hline KA018 & Pseudomonas otitidis (2) & MM2-3-1 & $4 / 11$ & 5 & 2 & 1 & 0 & 6 & 3 & 3 & 45 \\
\hline KA019 & & MP1-1-4 & $4 / 21$ & 4 & 0 & 1 & 1 & 4 & 2 & 0 & 26 \\
\hline KA020 & & MP1-2-1 & $10 / 30$ & 1 & 1 & 1 & 0 & 5 & 2 & 1 & 22 \\
\hline KA021 & Pseudomonas corrugata (1) & MP1-2-2 & $10 / 13$ & 2 & 2 & 1 & 0 & 4 & 2 & 0 & 28 \\
\hline KA022 & & MP1-3-1 & $10 / 13$ & 6 & 2 & 1 & 0 & 6 & 2 & 0 & 44 \\
\hline KA023 & & MP1-3-2 & $10 / 13$ & 6 & 2 & 1 & 0 & 6 & 2 & 0 & 46 \\
\hline
\end{tabular}


Table 2. Cont.

\begin{tabular}{|c|c|c|c|c|c|c|c|c|c|c|c|}
\hline \multicolumn{4}{|c|}{ Strain information } & \multirow[b]{2}{*}{ Antagonism } & \multirow{2}{*}{$\begin{array}{l}\text { Nutrient } \\
\text { allocation }\end{array}$} & \multirow{2}{*}{$\begin{array}{l}\text { Exoenzyme } \\
\text { activity }\end{array}$} & \multirow{2}{*}{$\begin{array}{l}\text { AB gene } \\
\text { detection }\end{array}$} & \multirow[b]{2}{*}{ Stress } & \multirow{2}{*}{$\begin{array}{l}\text { Plant } \\
\text { assay }\end{array}$} & \multirow{2}{*}{$\begin{array}{l}\text { Surface } \\
\text { activity }\end{array}$} & \multirow{2}{*}{$\begin{array}{l}\text { Score } \\
\text { values }\end{array}$} \\
\hline Abb. & Species (risk group) & $\begin{array}{c}\text { Strain } \\
\text { number }^{\text {a }}\end{array}$ & $\begin{array}{c}\text { ARDRA/ } \\
\text { BOX group }\end{array}$ & & & & & & & & \\
\hline KA024 & & MP1-3-3 & $12 / 23$ & 6 & 2 & 1 & 0 & 6 & 1 & 0 & 43 \\
\hline KA025 & & MP1-3-4 & $10 / 14$ & 4 & 1 & 1 & 1 & 5 & 3 & 0 & 33 \\
\hline KA026 & & MP2-1-2 & $10 / 13$ & 3 & 1 & 1 & 0 & 4 & 2 & 1 & 30 \\
\hline KA027 & Pseudomonas kilonensis (1) & MP2-1-4 & $10 / 13$ & 3 & 1 & 1 & 0 & 6 & 2 & 0 & 34 \\
\hline KA028 & & MP2-2-2 & $10 / 13$ & 4 & 2 & 1 & 0 & 7 & 3 & 0 & 44 \\
\hline KA029 & & RF1-1-1 & $10 / 4$ & 5 & 2 & 1 & 0 & 5 & 3 & 0 & 37 \\
\hline KA030 & Pseudomonas asplenii (1p) & RF1-2-2 & $3 / 24$ & 6 & 1 & 1 & 1 & 3 & 1 & 0 & 33 \\
\hline KA031 & & RF1-3-3 & $1 / 38$ & 2 & 0 & 1 & 0 & 1 & 1 & 1 & 14 \\
\hline KA032 & Pseudomonas asplenii (1p) & RF2-1-5 & $10 / 4$ & 2 & 1 & 2 & 0 & 1 & 2 & 0 & 19 \\
\hline KA033 & & RF2-3-3 & $10 / 13$ & 6 & 1 & 0 & 1 & 4 & 1 & 0 & 33 \\
\hline KA034 & Pseudomonas brenneri (1) & RF2-3-4 & $10 / 37$ & 5 & 1 & 1 & 0 & 5 & 1 & 0 & 32 \\
\hline KA035 & & RF2-3-5 & $10 / 4$ & 2 & 2 & 1 & 0 & 4 & 3 & 1 & 30 \\
\hline KA036 & $\begin{array}{l}\text { Pseudomonas brassicacearum subsp. } \\
\text { brassicacearum (1) }\end{array}$ & RM1-1-3 & $10 / 9$ & 4 & 1 & 0 & 1 & 3 & 1 & 0 & 26 \\
\hline KA037 & Pseudomonas kilonensis (1) & RM1-1-4 & $10 / 13$ & 4 & 1 & 0 & 0 & 5 & 3 & 0 & 32 \\
\hline KA038 & Pseudomonas otitidis (1) & RM1-3-1 & $4 / 11$ & 6 & 1 & 1 & 0 & 7 & 3 & 0 & 47 \\
\hline KA039 & & RM2-1-2 & $5 / 1$ & 5 & 0 & 0 & 1 & 5 & 3 & 0 & 30 \\
\hline KA040 & & RM2-1-5 & $10 / 19$ & 6 & 0 & 0 & 1 & 5 & 2 & 1 & 33 \\
\hline KA041 & & RM2-2-2 & $5 / 1$ & 1 & 1 & 0 & 0 & 4 & 3 & 1 & 26 \\
\hline KA042 & Pantoea sp. & RM2-2-4 & $5 / 40$ & 5 & 0 & 0 & 0 & 6 & 3 & 1 & 36 \\
\hline KA043 & & RM2-3-1 & $10 / 9$ & 4 & 0 & 0 & 0 & 6 & 2 & 1 & 30 \\
\hline KA044 & & RM2-3-3 & $10 / 9$ & 5 & 1 & 0 & 0 & 5 & 3 & 0 & 35 \\
\hline KA045 & & RP1-1-1 & $5 / 33$ & 5 & 1 & 0 & 0 & 5 & 2 & 0 & 32 \\
\hline KA046 & & RP1-1-3 & $10 / 14$ & 1 & 1 & 0 & 0 & 6 & 3 & 1 & 32 \\
\hline KA047 & & RP1-2-1 & $10 / 14$ & 3 & 2 & 1 & 0 & 5 & 3 & 0 & 32 \\
\hline KA048 & Pseudomonas rhodesiae (1) & RP1-2-2 & $10 / 14$ & 2 & 3 & 1 & 0 & 5 & 2 & 0 & 31 \\
\hline KA049 & & RP1-3-1 & $10 / 29$ & 4 & 3 & 1 & 0 & 5 & 4 & 0 & 38 \\
\hline
\end{tabular}


Table 2. Cont

\begin{tabular}{|c|c|c|c|c|c|c|c|c|c|c|c|}
\hline \multicolumn{4}{|c|}{ Strain information } & \multirow[b]{2}{*}{ Antagonism } & \multirow{2}{*}{$\begin{array}{l}\text { Nutrient } \\
\text { allocation }\end{array}$} & \multirow{2}{*}{$\begin{array}{c}\text { Exoenzyme } \\
\text { activity }\end{array}$} & \multirow{2}{*}{$\begin{array}{l}\text { AB gene } \\
\text { detection }\end{array}$} & \multirow[b]{2}{*}{ Stress } & \multirow{2}{*}{$\begin{array}{l}\text { Plant } \\
\text { assay }\end{array}$} & \multirow[b]{2}{*}{$\begin{array}{l}\text { Surface } \\
\text { activity }\end{array}$} & \multirow{2}{*}{$\begin{array}{l}\text { Score } \\
\text { values }\end{array}$} \\
\hline Abb. & Species (risk group) & $\begin{array}{c}\text { Strain } \\
\text { number }^{\text {a }}\end{array}$ & $\begin{array}{c}\text { ARDRA/ } \\
\text { BOX group }\end{array}$ & & & & & & & & \\
\hline KA050 & & RP1-3-3 & $5 / 12$ & 4 & 2 & 0 & 0 & 3 & 3 & 1 & 32 \\
\hline KA051 & Pseudomonas asplenii (1p) & RP1-3-4 & $10 / 4$ & 1 & 2 & 1 & 0 & 5 & 2 & 1 & 28 \\
\hline KA052 & & RP2-1-3 & $10 / 14$ & 6 & 0 & 1 & 1 & 3 & 2 & 0 & 33 \\
\hline KA053 & & RP2-2-2 & $14 / 39$ & 3 & 2 & 1 & 0 & 5 & 3 & 0 & 31 \\
\hline KA054 & & RP2-2-4 & $10 / 14$ & 1 & 0 & 2 & 0 & 4 & 3 & 0 & 24 \\
\hline KA055 & & SF1-1-1 & $10 / 14$ & 4 & 3 & 1 & 0 & 5 & 3 & 0 & 37 \\
\hline KA056 & & SF1-1-4 & $5 / 8$ & 2 & 2 & 1 & 0 & 5 & 4 & 0 & 32 \\
\hline KA057 & Serratia proteamaculans (2) & SF1-3-1 & $11 / 17$ & 1 & 2 & 0 & 0 & 5 & 3 & 1 & 28 \\
\hline KA058 & Yersinia kristensenii (2) & SF2-1-2 & $9 / 35$ & 2 & 2 & 1 & 0 & 6 & 4 & 1 & 40 \\
\hline KA059 & & SF2-2-1 & $9 / 35$ & 3 & 2 & 1 & 0 & 5 & 2 & 1 & 34 \\
\hline KA060 & Pseudomonas poae (1) & SF2-2-2 & $10 / 36$ & 2 & 1 & 1 & 0 & 5 & 3 & 0 & 30 \\
\hline KA061 & & SF2-2-4 & $10 / 37$ & 3 & 2 & 1 & 0 & 6 & 2 & 0 & 38 \\
\hline KA062 & & SF2-3-1 & $10 / 32$ & 1 & 1 & 1 & 0 & 5 & 3 & 0 & 25 \\
\hline KA063 & Pseudomonas veronii (1) & SM1-1-2 & $10 / 30$ & 2 & 1 & 0 & 0 & 5 & 3 & 0 & 24 \\
\hline KA064 & Pseudomonas koreensis (1) & SM1-1-4 & $10 / 20$ & 3 & 2 & 1 & 0 & 2 & 3 & 1 & 26 \\
\hline KA065 & Pseudomonas kilonensis (1) & SM1-2-4 & $10 / 13$ & 5 & 1 & 0 & 1 & 5 & 3 & 1 & 37 \\
\hline KA066 & Pseudomonas brenneri (1) & SM1-3-1 & $10 / 34$ & 4 & 2 & 1 & 0 & 5 & 2 & 0 & 38 \\
\hline KA067 & Pseudomonas otitidis (2) & SM1-3-2 & $10 / 34$ & 3 & 2 & 1 & 0 & 5 & 3 & 1 & 35 \\
\hline KA068 & & SM1-3-4 & $10 / 34$ & 6 & 2 & 1 & 0 & 5 & 4 & 1 & 44 \\
\hline KA069 & Enterobacter cowanii (2) & SM2-1-2 & $4 / 31$ & 3 & 2 & 1 & 0 & 5 & 1 & 0 & 29 \\
\hline KA070 & Pseudomonas veronii (1) & SM2-2-4 & $4 / 30$ & 3 & 0 & 0 & 0 & 5 & 4 & 1 & 33 \\
\hline KA071 & & SM2-3-1 & $7 / 31$ & 4 & 1 & 1 & 0 & 5 & 2 & 1 & 31 \\
\hline KA072 & & SM2-3-2 & $12 / 42$ & 4 & 0 & 1 & 0 & 5 & 3 & 0 & 28 \\
\hline KA073 & & SP1-1-1 & $2 / 41$ & 4 & 2 & 1 & 0 & 6 & 2 & 0 & 36 \\
\hline KA074 & Pseudomonas tremae (1) & SP1-1-3 & $18 / 27$ & 3 & 2 & 0 & 0 & 3 & 3 & 1 & 32 \\
\hline KA075 & & SP1-1-4 & $6 / 28$ & 2 & 2 & 1 & 1 & 4 & 6 & 0 & 37 \\
\hline KA076 & Serratia proteamaculans (2) & SP1-3-1 & $8 / 22$ & 3 & 0 & 1 & 0 & 5 & 2 & 1 & 27 \\
\hline KA077 & & SP2-1-2 & $16 / 14$ & 4 & 3 & 1 & 0 & 5 & 6 & 0 & 47 \\
\hline
\end{tabular}


Table 2. Cont

\begin{tabular}{|c|c|c|c|c|c|c|c|c|c|c|c|}
\hline \multicolumn{4}{|c|}{ Strain information } & \multirow[b]{2}{*}{ Antagonism } & \multirow[b]{2}{*}{$\begin{array}{l}\text { Nutrient } \\
\text { allocation }\end{array}$} & \multirow[b]{2}{*}{$\begin{array}{c}\text { Exoenzyme } \\
\text { activity }\end{array}$} & \multirow[b]{2}{*}{$\begin{array}{l}\text { AB gene } \\
\text { detection }\end{array}$} & \multirow[b]{2}{*}{ Stress } & \multirow[b]{2}{*}{$\begin{array}{l}\text { Plant } \\
\text { assay }\end{array}$} & \multirow[b]{2}{*}{$\begin{array}{l}\text { Surface } \\
\text { activity }\end{array}$} & \multirow[b]{2}{*}{$\begin{array}{l}\text { Score } \\
\text { values }\end{array}$} \\
\hline Abb. & Species (risk group) & $\begin{array}{c}\text { Strain } \\
\text { number }\end{array}$ & $\begin{array}{c}\text { ARDRA/ } \\
\text { BOX group }\end{array}$ & & & & & & & & \\
\hline KA078 & Pseudomonas putida (1) & SP2-2-2 & $15 / 13$ & 3 & 1 & 1 & 0 & 5 & 5 & 1 & 33 \\
\hline KA079 & & SP2-2-3 & $16 / 13$ & 3 & 2 & 1 & 0 & 5 & 7 & 0 & 42 \\
\hline KA080 & Pseudomonas rhodesiae (1) & SP2-3-3 & $16 / 14$ & 5 & 3 & 1 & 0 & 4 & 4 & 1 & 41 \\
\hline KA081 & Pseudomonas rhodesiae (1) & SP2-3-4 & $16 / 14$ & 3 & 3 & 1 & 0 & 2 & 4 & 1 & 29 \\
\hline KA082 & Pseudomonas poae (1) & ZF1-2-4 & $16 / 26$ & 0 & 2 & 1 & 0 & 4 & 2 & 1 & 26 \\
\hline KA083 & & ZF2-1-1 & $15 / 15$ & 1 & 0 & 0 & 0 & 5 & 2 & 0 & 20 \\
\hline KA084 & Pseudomonas corrugata (1) & ZF2-1-2 & $16 / 13$ & 5 & 2 & 1 & 0 & 5 & 1 & 1 & 38 \\
\hline KA085 & & ZF2-1-4 & $16 / 2$ & 2 & 3 & 0 & 0 & 5 & 1 & 1 & 28 \\
\hline KA086 & & ZF2-2-1 & $16 / 30$ & 1 & 2 & 1 & 0 & 3 & 2 & 1 & 26 \\
\hline KA087 & & ZF2-2-3 & $15 / 24$ & 1 & 1 & 0 & 0 & 0 & 3 & 1 & 10 \\
\hline KA088 & & ZF2-3-2 & $16 / 5$ & 1 & 0 & 2 & 0 & 5 & 4 & 1 & 29 \\
\hline KA089 & & ZF2-3-4 & $16 / 32$ & 1 & 1 & 1 & 0 & 5 & 2 & 1 & 27 \\
\hline KA090 & Microbacterium xylanilyticum (1) & ZM1-1-3 & $15 / 25$ & 1 & 0 & 1 & 0 & 4 & 4 & 1 & 27 \\
\hline KA091 & Chryseobacterium wanjuense (1) & ZM2-1-1 & $17 / 18$ & 2 & 1 & 1 & 0 & 7 & 1 & 0 & 32 \\
\hline KA092 & Pseudomonas kilonensis (1) & ZM2-1-2 & $16 / 13$ & 5 & 1 & 2 & 0 & 5 & 3 & 0 & 37 \\
\hline KA093 & Pseudomonas otitidis (1) & ZM2-2-3 & $16 / 11$ & 2 & 1 & 1 & 0 & 4 & 4 & 0 & 29 \\
\hline KA094 & Pseudomonas corrugata (1) & ZM2-3-1 & $16 / 13$ & 7 & 3 & 1 & 0 & 4 & 4 & 1 & 45 \\
\hline KA095 & Rhodanobacter fulvus (1) & ZM2-3-2 & $17 / 43$ & 2 & 1 & 0 & 0 & 3 & 4 & 1 & 26 \\
\hline KA096 & Pseudomonas kilonensis (1) & ZM2-3-4 & $16 / 13$ & 5 & 2 & 1 & 0 & 5 & 2 & 0 & 34 \\
\hline KA097 & & ZP1-1-3 & $16 / 26$ & 2 & 1 & 1 & 1 & 5 & 3 & 1 & 29 \\
\hline KA098 & & ZP1-1-4 & $16 / 6$ & 1 & 2 & 0 & 0 & 5 & 4 & 1 & 29 \\
\hline KA099 & Pseudomonas kilonensis (1) & ZP1-2-2 & $16 / 13$ & 5 & 2 & 1 & 0 & 4 & 1 & 1 & 33 \\
\hline KA100 & & ZP1-2-4 & $16 / 18$ & 2 & 1 & 0 & 0 & 6 & 3 & 1 & 28 \\
\hline KA101 & Pseudomonas kilonensis (1) & ZP1-3-1 & $16 / 13$ & 5 & 3 & 1 & 0 & 4 & 3 & 0 & 37 \\
\hline KA102 & Pseudomonas kilonensis (1) & ZP1-3-2 & $16 / 13$ & 6 & 3 & 1 & 0 & 4 & 4 & 0 & 39 \\
\hline KA103 & Pseudomonas kilonensis (1) & ZP1-3-3 & $16 / 13$ & 6 & 3 & 1 & 0 & 4 & 1 & 0 & 34 \\
\hline KA104 & & ZP1-3-4 & $15 / 10$ & 3 & 2 & 0 & 1 & 6 & 3 & 0 & 35 \\
\hline KA105 & Bacillus anthracis (3) & $\mathrm{ZP} 2-1-3$ & n.d./44 & 2 & 1 & 0 & 0 & 7 & 2 & 1 & 30 \\
\hline
\end{tabular}


To select a diverse group of bacteria and also to maintain quality control for further application processes, bacteria were genetically characterized using fingerprints from ARDRA and BOX-PCR. The antagonists were grouped according to the similarity index at 70\% into 44 various BOX groups (Figure S1, Table 2). One isolate from the most dominant group was sequenced, and species identification was determined after BLASTn analysis. Group 13 included the highest number of isolates (29), which were assigned to Pseudomonas kilonensis/P. corrugata.

Registration procedures inhibit the progress of the commercial application of bioproducts, due to their intensive costs and time requirements [24]. To improve positive product evaluation, potential human pathogenicity has to be identified and excluded from further work in advance. In an extensive study, a broad range of BCAs, pathogens and plant-associated bacteria were used in a rapid and inexpensive slow killing assay using Caenorhabditis elegans to estimate the risk of bacteria to human health [25]. In the current study, we analyzed the potential of the SPAs to grow at $37^{\circ} \mathrm{C}$, the human body temperature, and sequenced the partial 16S rRNA genes of antagonists. A high percentage (81.0 to $81.9 \%$ ) of the bacteria, including the highest scored bacteria, RM1-3-1 (KA38), SP2-1-2 (KA77) and MP1-3-2 (KA23), were able to grow at $37^{\circ} \mathrm{C}$; however, they were identified as Pseudomonas species in risk group 1. Identified species with potential human pathogenicity (risk group two and higher; Table 2) will be excluded from the next step of analysis in both greenhouse and field trials. Sequence similarities based on the 16S rRNA genes were visualized in a phylogenetic tree (Figure 4). The biggest ARDRA groups (10 with 46 isolates, 16 with 21 isolates) with a similarity index of $80 \%$ and BOX groups (Table 2) from the diverse Pseudomonas clusters were dominated by P. kilonensis and P. corrugata, and minorly represented by $P$. brassicacearum subsp. brassicacearum and P. poae species. Pseudomonas species are one of the primary bacteria that succeed in applications against phytopathogens [26].

To link the dominant bacteria from cultivation to fingerprints, the randomly picked isolates were included within the microbial fingerprint analysis ladder (Figure 1). Particular bands of the ladders were also found within the community patterns, e.g., Rhodanobacter fulvus strain ZM2-3-2 (KA95, score 26) isolated from the moss treated sugar beet samples was found within the sugar beet fingerprints with the same migration distance. In the moss-treated oilseed rape fingerprints, the bands of the cultivated bacteria, Arthrobacter nicotinovorans MM1-3-4 (KA14, score 27), Pseudomonas brassicacearum subsp. brassicacearum RM1-1-3 (KA36, score 26), Pseudomonas otitidis RM1-3-1 (KA38, score 47) and Pantoea sp. RM2-2-4 (KA42, score 36), were detected at the same migration level. The bacteria with the highest scores, i.e., Pseudomonas otitidis RM1-3-1 (KA38) or Pantoea sp. RM2-2-4 (KA42), were not part of the dominant community (weak SSCP band signal), but were successfully captured by the bait plants and selected through the appropriate screenings. However, in addition to the directed selection, those bacteria already present in the host-associated microbial community could also be isolated, e.g., Pseudomonas veronii strain SM1-1-2 (KA63, score 24). Directed selection is the first step in SPA isolation; however, more importantly, it is the evaluation of isolates in regards to their role as mediators of stress protection. The first results from evaluation under greenhouse conditions are promising. 
Figure 4. Phylogenetic composition of the antagonistic community selected by bait plants. The tree is comprised of 47 partial 16S rRNA gene sequences. Neighbor-joining phylogenetic tree (maximum composite likelihood method) was constructed with both one representative sequence per major species and the nearest reference sequences (accession numbers in brackets). The reliability of the tree topology was evaluated using bootstrap analysis with 10,000 re-samplings. Color code: rhizosphere samples from maize, yellow/orange/red; oilseed rape, green shades; sorghum, blue shades; sugar beet, purple shades. Sequences of MF2-1-4 (KA3) and ZM1-1-3 (KA90) were excluded from MEGA5 analysis due to their short sequence length.

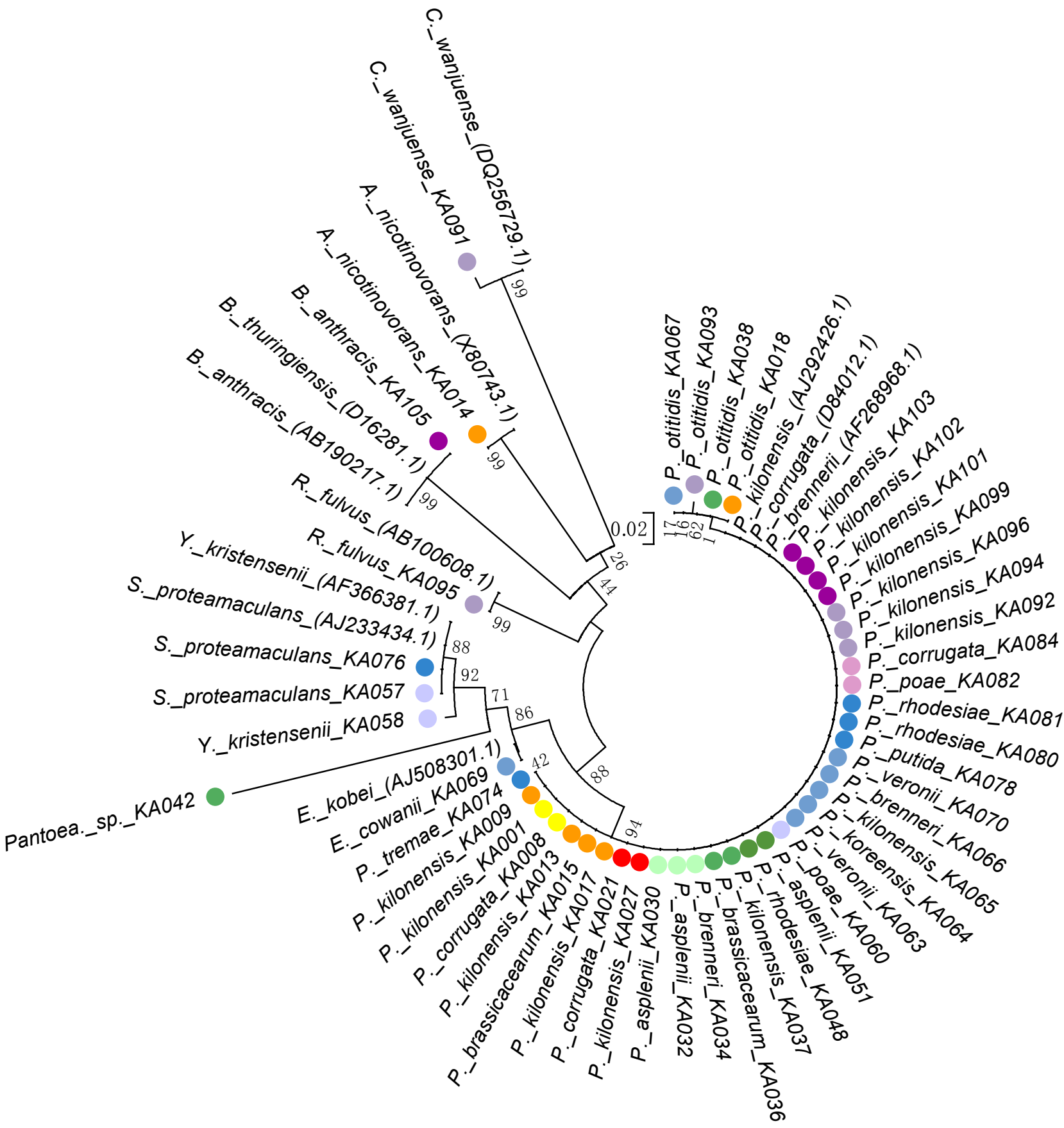




\section{Experimental Section}

\subsection{Experimental Design for Isolation and Characterization of Stress Protecting Bacteria}

The experimental design is comprised of the moss, Sphagnum magellanicum (M), the lichen, Solorina crocea $(\mathrm{F})$, and the Alpine Primula vulgaris $(\mathrm{P})$ for the screening of stress tolerance mediating bacteria (Tables S1 and S2). To ensure that we captured the most diverse microbial community possible, entire individuals of the two collected plant and lichen species were suspended in sterile $0.85 \%$ sodium chloride for three min in a stomacher laboratory blender (BagMixer; Interscience, St. Nom, France) in four replicates ( $P$. vulgaris, only 3 replicates). Aliquots of the suspensions were diluted and spread on R2A (the minimal medium to isolate universal bacteria bacteria) for determination of abundances and isolation after incubation for five days at room temperature. For directed selection of microorganisms over the rhizosphere of bait plants (maize, M; oilseed rape, R; sorghum, S; sugar beet, Z), the seeds were primed for four $\mathrm{h}$ in two different suspensions per material with three replicates at room temperature under agitation. Seeds were planted in $200-\mathrm{mL}$ pots filled with soil and subsequently cultivated (Gramoflor Profi-Substrat, Topfpikier M + Clay + Fe, $\mathrm{pH}\left(\mathrm{CaCl}_{2}\right)$ 5.8, Garten Bau Centrum, Kalsdorf, Austria, containing white and black peat with moist clay, $90 \mathrm{~kg} \mathrm{~m}^{3}$, PG-Mix $1.0 \mathrm{~kg} \mathrm{~m}^{3}$ (Greenworld, Wels, Austria), Radigen ${ }^{\circledR} 50 \mathrm{~g} \mathrm{~m}^{3}$ (Terraflor, Iserlohn, Germany)) and incubated under greenhouse conditions ( $12 \mathrm{~h}$ light/dark at $15{ }^{\circ} \mathrm{C} / 10{ }^{\circ} \mathrm{C}, 60 \%$ humidity). Two weeks after germination, when plants developed the first leaves, the rhizospheres were harvested, and microbial communities were suspended as described above. Dilutions were spread on different media as mentioned above. Host-adapted and stress protecting agents (SPAs) were randomly selected (30 per plant and treatment) and characterized. For cultivation-independent analysis, four milliliters of each suspension were centrifuged $(16,500 \times g$, $20 \mathrm{~min}, 4^{\circ} \mathrm{C}$ ) and stored at $-70^{\circ} \mathrm{C}$. Total community DNA was extracted using the FastDNA SPIN Kit for Soil (MP Biomedicals, Solon, USA) according to the manufacturer's protocol and used for fingerprint analysis. Bacterial DNA from single isolates was extracted according to Zachow et al. [27]. Bacterial isolates were labeled according to their host, treatment, replicate and continuous numbering; e.g., the third selected bacterial isolate selected from maize treated with the first extract of lichens in the second replicate is MF1-2-3; for practical laboratory work, a consecutive numbering was used, e.g., KA2.

\subsection{Molecular Analysis}

Profiling of microbial communities was carried out with single strand conformational polymorphism analysis (SSCP) on a PhorU2 apparatus (Ingeny, Goes, The Netherlands) and as described by Köberl et al. [7]. 16S rRNA genes of bacteria were amplified using a universal primer according to the protocol described in Zachow et al. [27], and three independent replicates were included for each sample. Bacterial isolates were screened for genes encoding for nitrogenase $(n i f \mathrm{H})$; fragments were amplified using a nested approach with primer pairs nifH3/19F and nifH11/nifH22 [28]. Genes for potential antibiotic production (2,4-diacetylphloroglucinol phlD, pyrrolnitrin prnD) were amplified according to Raaijmakers et al. [29] and de Souza and Raaijmakers [30]. Amplicons were compared with positive control strains containing the corresponding genes. Amplified ribosomal RNA gene restriction analysis (ARDRA) by applying the restriction endonuclease, HhaI (MP Biomedicals, 
Eschwege, Germany), to the $1492 \mathrm{r}$ and $27 \mathrm{f}$ fragment was performed according to Zachow et al. [27]. ARDRA patterns were used to group isolates at the genus level, and isolates displaying similar patterns were further analyzed using the PCR-dependent fingerprinting method with repetitive BOX-elements, according to Martin et al. [31]. Antagonists with unique BOX patterns and a high evaluation score (see part 2.3) were identified by partial $16 \mathrm{~S}$ rRNA gene sequence analysis, according to Berg et al. [32]. The neighbor-joining phylogenetic tree was constructed using Molecular Evolutionary Genetics Analysis version 5 (MEGA5) [33].

\subsection{Stress Confrontation Assays}

Overnight cultures (ONC) grown in nutrient broth II (NBII, Sifin, Germany) were used as inoculum $(10 \mu \mathrm{L})$ for all bacterial assays. For osmolarity stress, bacteria were cultivated in $1.5 \mathrm{~mL}$ tubes filled with $700 \mu \mathrm{L}$ NBII with various sodium chloride concentrations ( $1 \%$ steps, $0 \%-20 \%$ ) and incubated at $30{ }^{\circ} \mathrm{C}$ for $48 \mathrm{~h}$ under agitation. For desiccation assays, $20 \mu \mathrm{L}$ of the ONCs were dried under sterile conditions. After $24 \mathrm{~h}$, bacteria were re-suspended with the same volume of NBII and dropped on nutrient broth II agar (NAII). For growth assays under different temperatures, bacteria were streaked onto NAII and R2A plates and incubated at $13{ }^{\circ} \mathrm{C}$ and $37{ }^{\circ} \mathrm{C}$. In reactive oxygen species stress assays, bacteria were cultivated in NBII amended with different tellurite $\left(1,3,5,7,9\right.$ and $\left.10 \mathrm{mg} \mathrm{mL}^{-1}\right)$ and $\mathrm{H}_{2} \mathrm{O}_{2}(100,300$, $500,700,900,1000,1300$ and $1500 \mathrm{mM}$ ) concentrations. The assays were performed in 96-well-plates filled with $195 \mu \mathrm{L}$ medium per well and $5 \mu \mathrm{L}$ ONC. In all assays, bacterial growth was visually evaluated after $24 \mathrm{~h}$ and $48 \mathrm{~h}$. Bacteria were cultivated in 40-mL Luria-Bertani medium (LB, per liter: tryptone $10 \mathrm{~g}$, yeast extract $5 \mathrm{~g}$, sodium chloride $10 \mathrm{~g}, \mathrm{pH} 7.0$ ) at $30^{\circ} \mathrm{C}$ to measure antioxidant activity in a radical stress assay with tetramethoxy azobismethylene quinone (TMAMQ). After $24 \mathrm{~h}$, cells were harvested $\left(2500 \times \mathrm{g}, 5 \mathrm{~min}, 4^{\circ} \mathrm{C}\right)$ and washed three times with the same volume of sterile $0.85 \%$ sodium chloride. The antioxidant activity of unstressed (control) and stressed isolates $\left(50{ }^{\circ} \mathrm{C}\right.$ for 6 min and 20 min) was measured with TMAMQ developed by Prasetyo et al. [34].

\subsection{Plant Pathogen Confrontation Assays}

The in vitro inhibition of Alternaria alternata Nees (A. a.), Botrytis cinerea Pers. (B. c.), Phytophthora infestans (Mont.) De Bary (P. i.), Rhizoctonia solani AG2-2IIIB Kühn (R. s. AG2) and AG4 (R. s. AG4), Sclerotinia sclerotiorum Sacc. (S. s.), Sclerotium rolfsii (Curzi) C.C.Tu and Kimbr. $(S . r$.) and Verticillium dahliae Kleb. $(V . d$.) was determined by a dual-culture assay on agar plates (fungi Waksman agar, oomycetes rye sucrose agar) and evaluated according to Berg et al. (2002) [32]. Zones of hyperparasitism or inhibition were measured after five days of incubation at $25{ }^{\circ} \mathrm{C}$, and assays were done for each plant in two replicates. Bursts of $P$. infestans zoospores were tested according to de Bruijn et al. [35], and only antagonistic bacteria were used for further characterizations.

\subsection{Exoenzyme Activity, Biosurfactants and Nutrient Allocation}

The exoenzyme activity of chitinase (beta-1,4-glucosamine polymer degradation), beta-glucanase and protease (casein degradation) was determined according to Grube et al. [12]. Surface activity of the ONCs, washed cells $\left(2500 \times \mathrm{g}, 5 \mathrm{~min}, 4{ }^{\circ} \mathrm{C}\right)$ and the supernatant was tested in a drop assay on a 
polystyrene lid and in a flat-bottomed 96-well plate saturated with mineral and paraffin oil, according to Jain et al. [36]. The assay was also done with toluol $(400 \mu \mathrm{L})$ mixed with the isolates $(400 \mu \mathrm{L})$ and incubated for one hour. For rhamnolipid (glycolipidic biosurfactants) detection on plates, methylene blue-containing plates were prepared containing per liter: 20 g peptone, $0.7 \mathrm{~g} \mathrm{KH}_{2} \mathrm{PO}_{4}, 0.9 \mathrm{~g} \mathrm{Na}_{2} \mathrm{HPO}_{4}$, $2 \mathrm{~g} \mathrm{NaNO}_{3}, 0.4 \mathrm{~g} \mathrm{MgSO}_{4} \cdot 7 \mathrm{H}_{2} \mathrm{O}, 0.1 \mathrm{~g} \mathrm{CaCl}_{2} \cdot 2 \mathrm{H}_{2} \mathrm{O}, 0.2 \mathrm{~g} \mathrm{CTAB}, 0.005 \mathrm{~g}$ methylene blue, $15 \mathrm{~g}$ agar and $2 \mathrm{~mL}$ trace elements solution $\left(2 \mathrm{~g} \mathrm{FeSO}_{4} \cdot 7 \mathrm{H}_{2} \mathrm{O}, 1.5 \mathrm{~g} \mathrm{MnSO}_{4} \cdot \mathrm{H}_{2} \mathrm{O}\right.$ and $0.6 \mathrm{~g}\left(\mathrm{NH}_{4}\right)_{6} \mathrm{MO}_{7} \mathrm{O}_{24}$ acidified with $37 \% \mathrm{HCl}$ ). Phosphate solubilization was tested according to Fürnkranz et al. [37].

\subsection{Bioassays}

The ability to produce indole-3-acetic acid (IAA) was tested for selected bacterial strains in a plate test according to Sawar and Kremer [38]. Nitrogen fixation and production of the ethylene precursor, 1-aminocyclopropane-1-carboxylic acid (ACC) deaminase, was tested according to Fürnkranz et al. [37]. Germination of primed seeds (seed number: M, 3; O, 20; S, 15; Z, 20; $1 \mathrm{~mL}$ ONC per well of a 24-well plate, $4 \mathrm{~h}$, room temperature, with agitation) was tested on wet filter paper in a 9-cm plate at $13{ }^{\circ} \mathrm{C}$. Germination was evaluated after three and six days in comparison to tap water, distilled water, sodium chloride and unprimed control. The experiment evaluated the first positive or negative impact of the bacteria on seed germination. Only treatments comparable or better than the controls were highly weighted. The best isolates from the pre-screening were tested in a large batch under the same conditions with four repetitions ( 25 seeds each plant, $10 \mathrm{~mL} \mathrm{ONC).}$

\subsection{Statistics}

To compare the SSCP fingerprints of the microbial communities, a computer-assisted cluster analysis was carried out using the GelCompar II $^{\circledR}$ software (Applied Math, Kortrijk, Belgium). Computer-assisted pattern analyses of molecular fingerprints of the communities resulted in correlation matrices that were then subjected to a significance test by applying $10^{4}$ random permutations of sample elements [39]. The relationships between samples taken from different treated crops were investigated.

\section{Conclusions}

The bait plant-based selection with subsequent stress protection screening assays resulted in target-adapted microorganisms. The resulting SPAs from this selection strategy are taxonomically highly diverse and belong primarily to the Pseudomonas group (e.g., P. asplenii, P. brassicacearum, P. corrugata, $P$. kilonensis, $P$. otitidis, $P$. poae). They (except plant and human pathogenic species) can be further implemented into commercial crop production for enhanced plant health and stress tolerance, as the first field results are promising, and the applied evaluation score allows for a flexible and adjustable constitution of SPAs independent of their supposed biotechnological application. In using different bait plants, environmental conditions or microenvironments other than the rhizosphere, new implementations can be discovered in regards to the changing climate conditions or different soil types, e.g., desert soil, soils with extreme $\mathrm{pH}$ values or contaminated soil. Furthermore, the system can be used to "fine tune" highly specific host plant species and even cultivars. 


\section{Author Contributions}

The following conceived of and guided the research and wrote the manuscript: Christin Zachow, Henry Müller, Ralf Tilcher, Christina Donat, Gabriele Berg. The following performed the laboratory experiments: Christin Zachow, Henry Müller.

\section{Acknowledgments}

The authors would like to thank Elisabeth Mara, Barbara Fetz, Monika Schneider-Trampitsch, Melanie Marek, Lisa Oberauner and Martina Köberl (all Graz University of Technology, Austria), for valuable technical assistance, and Margaret R. Starcher (Graz, Washington), for the English revision. The authors acknowledge the help of Endry Nugroho Prasetyo and Gibson S. Nyanhongo (both of Graz University of Technology, Austria) for providing support in antioxidant activity measurements. This work has been supported by a grant to Gabriele Berg from the Federal Ministry of Economy, Family and Youth (BMWFJ), the Federal Ministry of Traffic, Innovation and Technology (bmvit), the Styrian Business Promotion Agency, SFG, the Standortagentur Tirol and ZIT (Technology Agency of the City of Vienna) through the COMET Funding Program managed by the Austrian Research Promotion Agency, FFG.

\section{Conflicts of Interest}

The authors declare no conflict of interest.

\section{References}

1. Shaw, M.W. Preparing for changes in plant disease due to climate change. Plant Prot. Sci. 2009, 45, S3-S10.

2. Newton, A.C.; Johnson, S.N.; Gregory, P.J. Implications of climate change for diseases, crop yields and food security. Euphytica 2011, 179, 3-18.

3. Maheswari, M.; Yadav, S.K.; Shanker, A.K.; Kumar, M.A.; Venkateswarlu, B. Overview of Plant Stresses: Mechanisms, Adaptations and Research Pursuit. In Crop Stress and its Management: Perspectives and Strategies; Venkateswarlu, B., Shanker, A.K., Shanker, C., Maheswari, M., Eds.; Springer: New York, NY, USA, 2012; pp. 1-18.

4. Berendsen, R.L.; Pieterse, C.M.; Bakker, P.A. The rhizosphere microbiome and plant health. Trends Plant Sci. 2012, 17, 478-486.

5. Alavi, P.; Starcher, M.R.; Zachow, C.; Müller, H.; Berg, G. Root-microbe systems: the effect and mode of interaction of Stress Protecting Agent (SPA) Stenotrophomonas rhizophila DSM14405 ${ }^{\mathrm{T}}$. Front. Plant Sci. 2013, 4, 141, doi:10.3389/fpls.2013.00141.

6. Berg, G.; Smalla, K. Plant species and soil type cooperatively shape the structure and function of microbial communities in the rhizosphere. FEMS Microbiol. Ecol. 2009, 68, 1-13.

7. Köberl, M.; Müller, H.; Ramadan, E.M.; Berg, G. Desert farming benefits from microbial potential in arid soils and promotes diversity and plant health. PLoS One 2011, 6, e24452. 
8. Köberl, M.; Ramadan, E.M.; Roßmann, B.; Staver, C.; Fürnkranz, M.; Lukesch, B.; Grube, M.; Berg, G. Using Ecological Knowledge and Molecular Tools to Develop Effective and Safe Biocontrol Strategies. In Pesticides in the Modern World-Pests Control and Pesticides Exposure and Toxicity Assessment; Stoytcheva, M., Ed.; InTech e-book: Rijeka, Croatia, 2011; pp. 3-26.

9. Yang, J.; Kloepper, J.W.; Ryu, C.M. Rhizosphere bacteria help plants tolerate abiotic stress. Trends Plant Sci. 2009, 14, 1-4.

10. Opelt, K.; Chobot, V.; Hadacek, F.; Schönmann, S.; Eberl, L.; Berg, G. Investigations of the structure and function of bacterial communities associated with Sphagnum mosses. Environ. Microbiol. 2007, 91, 2795-2809.

11. Opelt, K.; Berg, C.; Berg, G. The bryophyte genus Sphagnum is a reservoir for powerful and extraordinary antagonists and potentially facultative human pathogens. FEMS Microbiol. Ecol. 2007, 61, 38-53.

12. Grube, M.; Cardinale, M.; de Castro, J.V., Jr.; Müller, H.; Berg, G. Species-specific structural and functional diversity of bacterial communities in lichen symbioses. ISME J. 2009, 3, 1105-1115.

13. Berg, G.; Hartenberger, K.; Liebminger, S.; Zachow, C. Antagonistic Endophytes from Mistletoes as Bio-resource to Control Plant as well as Clean Room Pathogens. In IOBC/WPRS Bulletin, Working Group "Biological Control of Fungal and Bacterial Plant Pathogens”; Pertot, I., Elad, Y., Gessler, C., Cini, A., Eds.; International Organisation for Biological Control (IOBC): Zurich, Switzerland, 2012; Volume 78, pp. 29-32.

14. Zachow, C.; Grosch, R.; Berg, G. Impact of biotic and a-biotic parameters on structure and function of microbial communities living on sclerotia of the soil-borne pathogenic fungus Rhizoctonia solani. Appl. Soil Ecol. 2011, 48, 193-200.

15. Amann, R.I.; Ludwig, W.; Schleifer, K.H. Phylogenetic identification and in situ detection of individual microbial cells without cultivation. Microbiol. Rev. 1995, 59, 143-169.

16. Schwieger, F.; Tebbe, C.C. A new approach to utilize PCR-single-strand conformation polymorphism for 16S rRNA gene-based microbial community analysis. Appl. Environ. Microbiol. 1998, 64, 4870-4876.

17. Smalla, K.; Oros-Sichler, M.; Milling, A.; Heuer, H.; Baumgarte, S.; Becker, R.; Neuber, G.; Kropf, S.; Ulrich, A.; Tebbe, C.C. Bacterial diversity of soils assessed by DGGE, T-RFLP and SSCP fingerprints of PCR-amplified 16S rRNA gene fragments: Do the different methods provide similar results? J. Microbiol. Methods 2007, 69, 470-479.

18. Bragina, A.; Oberauner-Wappis, L.; Halwachs, B.; Thallinger, G.G.; Müller, H.; Berg, G. The Sphagnum microbiome support greatly ecosystem functioning in bog ecosystems under extreme conditions. 2013, submitted.

19. Grosch, R.; Dealtry, S.; Schreiter, S.; Berg, G.; Mendonça-Hagler, L.; Smalla, K. Biocontrol of Rhizoctonia solani: Complex interaction of biocontrol strains, pathogen and indigenous microbial community in the rhizosphere of lettuce shown by molecular methods. Plant Soil 2012, 361, 343-357.

20. Hartmann, A.; Schmid, M.; van Tuinen, D.; Berg, G. Plant-driven selection of microbes. Plant Soil 2009, 321, 235-257. 
21. Bulgarelli, D.; Rott, M.; Schlaeppi, K.; Ver Loren van Themaat, E.; Ahmadinejad, N.; Assenza, F.; Rauf, P.; Huettel, B.; Reinhardt, R.; Schmelzer, E.; et al. Revealing structure and assembly cues for Arabidopsis root-inhabiting bacterial microbiota. Nature 2012, 488, 91-95.

22. Lundberg, D.S.; Lebeis, S.L.; Paredes, S.H.; Yourstone, S.; Gehring, J.; Malfatti, S.; Tremblay, J.; Engelbrektson, A.; Kunin, V.; del Rio, T.G.; et al. Defining the core Arabidopsis thaliana root microbiome. Nature 2012, A488(7409), 86-90.

23. Hartmann, A.; Rothballer, M.; Schmid, M. Lorenz Hiltner, a pioneer in rhizosphere microbial ecology and soil bacteriology research. Plant Soil 2008, 312, 7-14.

24. Ehlers, R.U. Regulation of Biological Control Agents; Springer: Dordrecht, The Netherlands, 2011.

25. Zachow, C.; Pirker, H.; Westendorf, C.; Tilcher, R.; Berg, G. The Caenorhabditis elegans assay: a tool to evaluate the pathogenic potential of bacterial biocontrol agents. Eur. J. Plant Pathol. 2009, $125,367-376$.

26. Loper, J.E.; Hassan, K.A.; Mavrodi, D.V.; Davis, E.W., II.; Lim, C.K.; Shaffer, B.T.; Elbourne, L.D.H.; Stockwell, V.O.; Hartney, S.L.; Breakwell, K.; et al. Comparative genomics of plant-associated Pseudomonas spp.: Insights into diversity and inheritance of traits involved in multitrophic interactions. PLoS Genet. 2012, 8, e1002784.

27. Zachow, C.; Tilcher, R.; Berg, G. Sugar beet-associated bacterial and fungal communities show a high indigenous antagonistic potential against plant pathogens. Microbial Ecol. 2008, 55, 119-129.

28. Yeager, C.M.; Kornosky, L.; Housman, C.; Grote, E.E.; Belnap, J.; Kuske, C.R. Diazotrophic community structure and function in two successional stages of biological soil crusts from the Colorado Plateau and Chihuahuan Desert. Appl. Environ. Microbiol. 2004, 70, 973-983.

29. Raaijmakers, J.M.; Weller, D.M.; Thomashow, L.S. Frequency of antibiotic-producing Pseudomonas spp. in natural environments. Appl. Environ. Microbiol. 1997, 63, 881-887.

30. De Souza, J.T.; Raaijmakers, J.M. Polymorphisms within the prnD and pltC genes from pyrrolnitrin and pyoluteorin-producing Pseudomonas and Burkholderia spp. FEMS Microbiol. Ecol. 2003, 43, 21-34.

31. Martin, B.; Humbert, O.; Camara, M.; Guenzi, E.; Walker, J.; Mitchell, T.; Andrew, P.; Prudhomme, M.; Alloing, G.; Hakenbeck, R.; Morrison, D.A.; Boulnois, G.J.; Claverys, J.P. A highly conserved repeated DNA element located in the chromosome of Streptococcus pneumonia. Nucleic Acids Res. 1992, 20, 3479-3483.

32. Berg, G.; Roskot, N.; Steidle, A.; Eberl, L.; Zock, A.; Smalla, K. Plant-dependent genotypic and phenotypic diversity of antagonistic rhizobacteria isolated from different Verticillium host plants. Appl. Environ. Microbiol. 2002, 68, 3328-3338.

33. Tamura, K.; Peterson, D.; Peterson, N.; Stecher, G.; Nei, M.; Kumar, S. MEGA5: Molecular evolutionary genetics analysis using maximum likelihood, evolutionary distance, and maximum parsimony methods. Mol. Biol. Evol. 2011, 28, 2731-2739.

34. Prasetyo, E.N.; Kudanga, T.; Steiner, W.; Murkovic, M.; Wonisch, W.; Nyanhongo, G.S.; Gübitz, G.M. Cellular and plasma antioxidant activity assay using tetramethoxy azobismethylene quinone. Free Radic. Biol. Med. 2010, 49, 1205-1211.

35. De Bruijn, I.; de Kock, M.J.D.; Yang, M.; de Waard, P.; van Beek, T.A.; Raaijmakers, J.M. Genome-based discovery, structure prediction and functional analysis of cyclic lipopeptide antibiotics in Pseudomonas species. Mol. Microbiol. 2007, 63, 417-428. 
36. Jain, D.K.; Lee, H.; Trevors, J.T. Effect of addition of Pseudomonas aeruginosa UG2 inocula or biosurfactants on biodegradation of selected hydrocarbons in soil. J. Ind. Microbiol. 1992, 10, 87-93.

37. Fürnkranz, M.; Müller, H.; Berg, G. Characterization of plant growth promoting bacteria from crops in Bolivia. J. Plant Dis. Prot. 2009, 116, 149-155.

38. Sawar, M.; Kremer, R.J. Determination of bacterially derived auxins using a microplate method. Lett. Appl. Microbiol. 1995, 20, 282-285.

39. Kropf, S.; Heuer, H.; Grüning, M.; Smalla, K. Significance test for comparing complex microbial community fingerprints using pairwise similarity measures. J. Microbiol. Methods 2004, 57, $187-195$.

\section{Supplementary}

Table S1. Sampling details: bioresources.

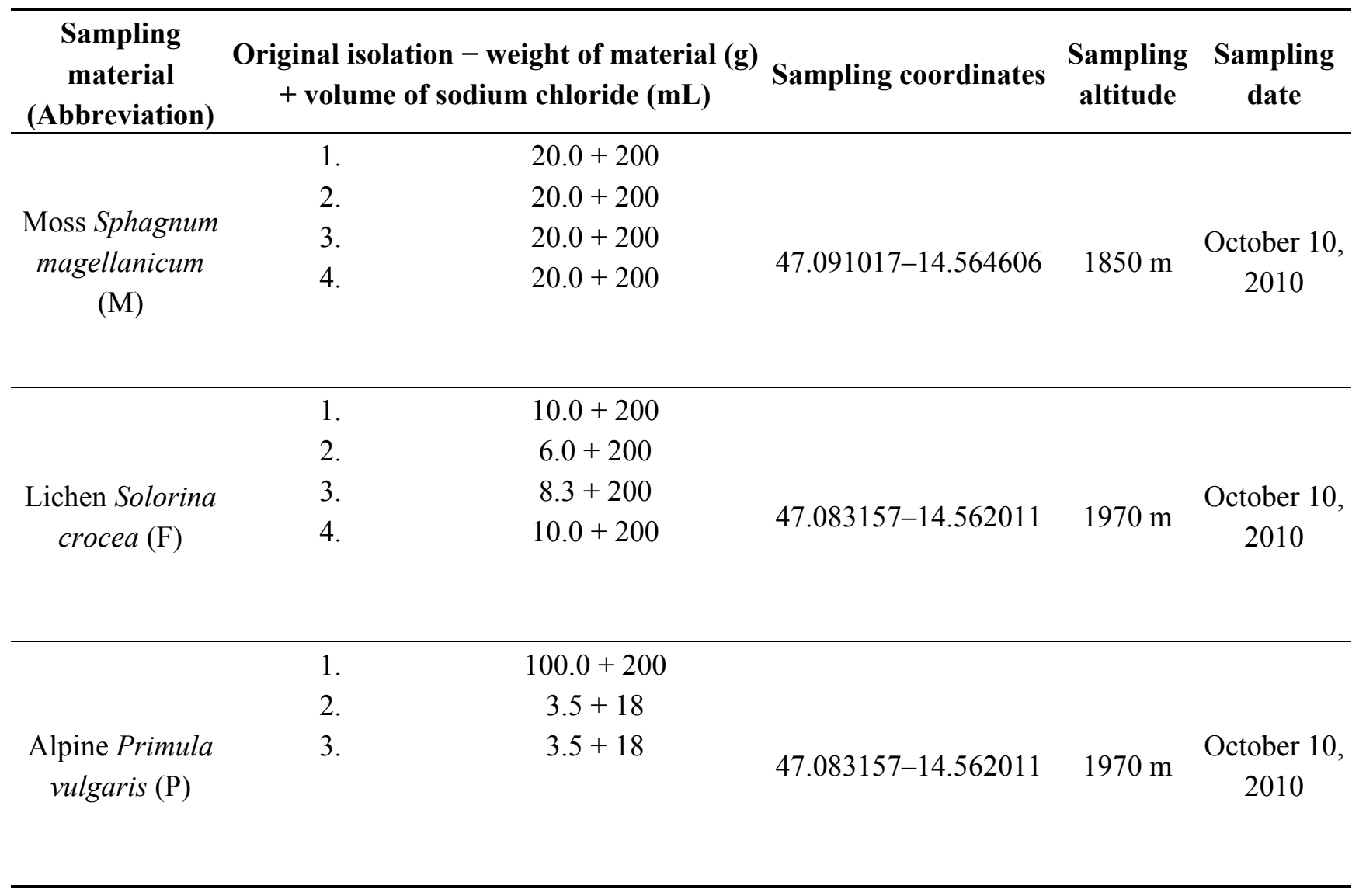


Table S2. Sampling details: treated plants. Treatments: C, control; F, lichen; M, moss; $\mathrm{P}$, primrose.

\begin{tabular}{|c|c|c|c|c|c|c|c|}
\hline \multirow{3}{*}{$\begin{array}{c}\text { Sampling material } \\
\text { (Abbreviation) }\end{array}$} & \multirow{3}{*}{ Treatment } & \multicolumn{6}{|c|}{$\begin{array}{l}\text { Bait plant isolation } \\
10.0 \mathrm{~mL} \text { sodium chloride }\end{array}$} \\
\hline & & \multicolumn{2}{|c|}{ Repetition 1} & \multicolumn{2}{|c|}{ Repetition 2} & \multicolumn{2}{|c|}{ Repetition 3} \\
\hline & & \# plants & $\mathrm{m}$ in $\mathrm{g}$ & \# plants & $\mathrm{m}$ in $\mathrm{g}$ & \# plants & $m$ in $g$ \\
\hline \multirow{7}{*}{ Maize (M) } & $\mathrm{C}$ & 4 & 8.27 & 4 & 17.78 & 3 & 15.71 \\
\hline & $\mathrm{F} 1$ & 4 & 15.95 & 3 & 11.21 & 4 & 15.53 \\
\hline & $\mathrm{F} 2$ & 4 & 16.41 & 4 & 11.33 & 3 & 11.87 \\
\hline & M1 & 4 & 19.09 & 4 & 23.94 & 3 & 14.62 \\
\hline & M2 & 4 & 14.19 & 3 & 13.76 & 3 & 14.85 \\
\hline & $\mathrm{P} 1$ & 4 & 19.58 & 4 & 13.96 & 4 & 17.90 \\
\hline & $\mathrm{P} 2$ & 3 & 11.56 & 4 & 18.45 & 3 & 12.58 \\
\hline \multirow{7}{*}{ Oilseed rape $(R)$} & $\mathrm{C}$ & 5 & 1.56 & 5 & 0.92 & 3 & 0.56 \\
\hline & $\mathrm{F} 1$ & 5 & 1.27 & 5 & 1.27 & 5 & 1.84 \\
\hline & $\mathrm{F} 2$ & 5 & 2.29 & 5 & 1.70 & 5 & 2.09 \\
\hline & M1 & 5 & 1.55 & 5 & 1.66 & 5 & 1.04 \\
\hline & M2 & 5 & 1.63 & 5 & 2.01 & 5 & 2.16 \\
\hline & $\mathrm{P} 1$ & 5 & 0.64 & 5 & 1.78 & 5 & 1.81 \\
\hline & $\mathrm{P} 2$ & 5 & 0.84 & 5 & 1.27 & 5 & 1.05 \\
\hline \multirow{7}{*}{ Sorghum (S) } & $\mathrm{C}$ & 2 & 0.57 & 5 & 1.75 & 3 & 1.08 \\
\hline & $\mathrm{F} 1$ & 4 & 1.59 & 3 & 0.86 & 3 & 1.12 \\
\hline & $\mathrm{F} 2$ & 2 & 1.63 & 3 & 1.77 & 2 & 0.89 \\
\hline & M1 & 3 & 1.60 & 4 & 2.05 & 3 & 2.50 \\
\hline & M2 & 3 & 1.53 & 4 & 1.29 & 4 & 1.74 \\
\hline & $\mathrm{P} 1$ & 2 & 2.11 & 3 & 0.82 & 4 & 1.68 \\
\hline & $\mathrm{P} 2$ & 4 & 1.68 & 5 & 1.75 & 4 & 1.67 \\
\hline \multirow{7}{*}{ Sugar beet (Z) } & $\mathrm{C}$ & 5 & 0.91 & 5 & 0.82 & 5 & 0.71 \\
\hline & $\mathrm{F} 1$ & 5 & 1.74 & 4 & 0.93 & 5 & 0.53 \\
\hline & $\mathrm{F} 2$ & 5 & 0.66 & 3 & 0.41 & 5 & 1.15 \\
\hline & M1 & 5 & 0.84 & 4 & 1.08 & 5 & 1.95 \\
\hline & M2 & 4 & 0.46 & 4 & 0.80 & 5 & 1.08 \\
\hline & $\mathrm{P} 1$ & 2 & 0.36 & 4 & 0.62 & 3 & 0.29 \\
\hline & $\mathrm{P} 2$ & 5 & 0.60 & 4 & 0.40 & 5 & 1.02 \\
\hline
\end{tabular}


Figure S1. BOX-PCR fingerprints of single isolates selected after priming seeds of maize, oilseed rape, sorghum and sugar beet with moss, lichen and primrose communities. UPGMA dendrogram obtained by GelCompar II ${ }^{\circledR}$; Pearson correlation with 5\% optimization.

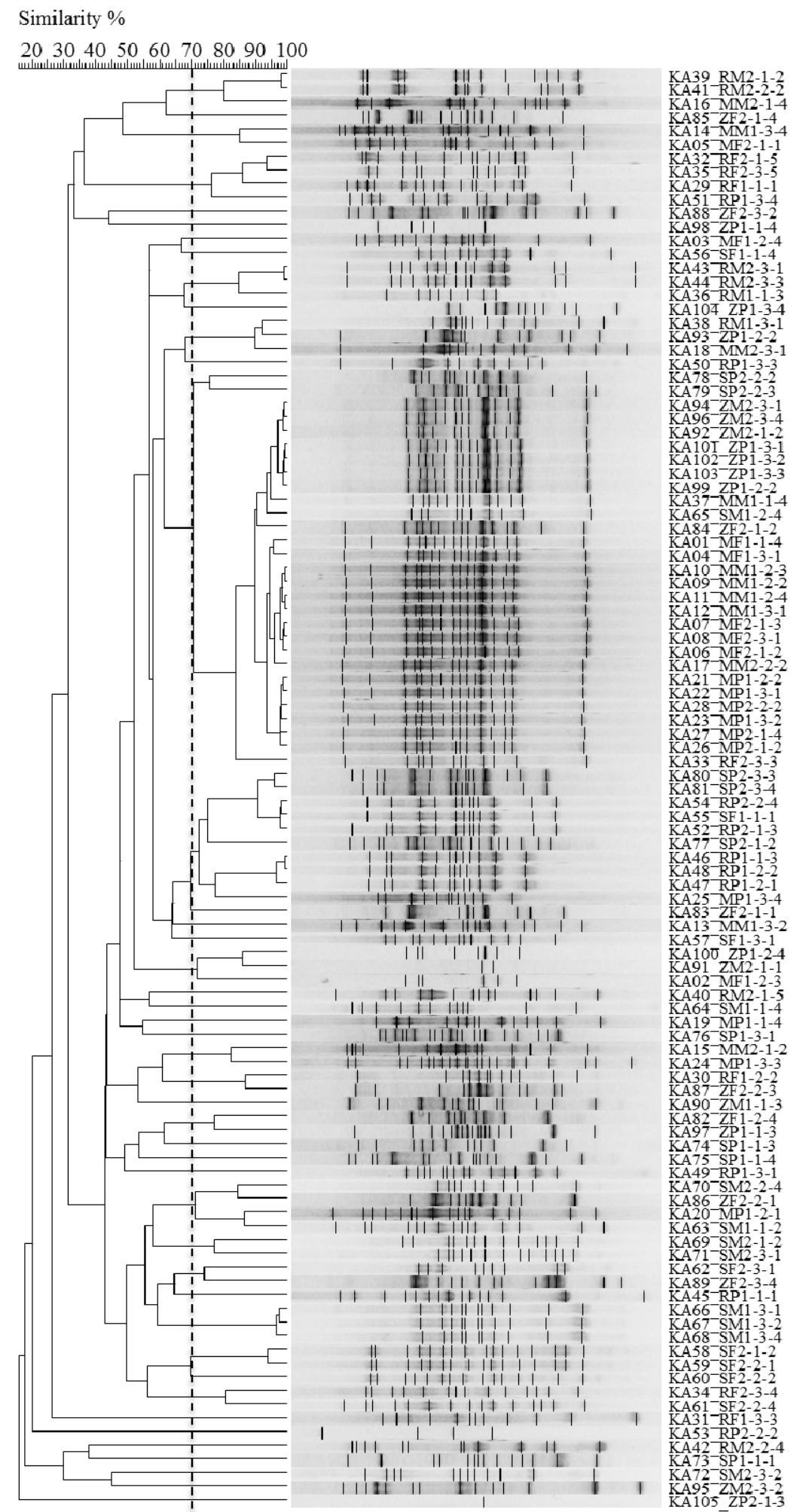

(C) 2013 by the authors; licensee MDPI, Basel, Switzerland. This article is an open access article distributed under the terms and conditions of the Creative Commons Attribution license (http://creativecommons.org/licenses/by/3.0/). 